\title{
The clinical utility of remote ischemic preconditioning in protecting against cardiac surgery-associated acute kidney injury: A pilot randomized clinical trial
}

\author{
Karolina Stokfisz ${ }^{1, A, B, D}$, Anna Ledakowicz-Polak ${ }^{1, A, D, E}$, Maciej Zagórski ${ }^{2, B, D}$, Sławomir Jander ${ }^{2, B}$, \\ Katarzyna Przybylak ${ }^{1, C}$, Marzenna Zielińska ${ }^{1, A, C, F}$ \\ ${ }^{1}$ Intensive Cardiac Therapy Clinic, Department of Invasive Cardiology and Electrocardiology, Medical University of Lodz, Poland \\ ${ }^{2}$ Cardiosurgery Clinic, Department of Cardiology and Cardiosurgery, Medical University of Lodz, Poland
}

\begin{abstract}
A - research concept and design; B - collection and/or assembly of data; $\mathrm{C}$ - data analysis and interpretation;
$D$ - writing the article; $E$ - critical revision of the article; $F$ - final approval of the article
\end{abstract}

Address for correspondence

Karolina Stokfisz

E-mail: stokfisz.karolina@gmail.com

Funding sources

None declared

Conflict of interest

None declared

Received on December 11, 2018

Reviewed on September 11, 2019

Accepted on September 25, 2019

Published online on February 24, 2020

Cite as

Stokfisz K, Ledakowicz-Polak A, Zagórski M, Jander S, Przybylak K, Zielińska M. The clinical utility of remote ischemic preconditioning in protecting against cardiac surgery-associated acute kidney injury: A pilot randomized clinical trial. Adv Clin Exp Med. 2020;29(2):189-196. doi:10.17219/acem/112610

DOI

10.17219/acem/112610

Copyright

Copyright by Author(s)

This is an article distributed under the terms of the

Creative Commons Attribution 3.0 Unported (CC BY 3.0)

(https://creativecommons.org/licenses/by/3.0/)

\begin{abstract}
Background. Cardiac surgery-associated acute kidney injury (CSA-AKI) is a well-known, serious complication and a well-recognized independent risk factor for higher morbidity and mortality among patients undergoing cardiac surgery.

Objectives. The aim of the study was to assess the efficacy of remote ischemic preconditioning (RIPC) in reducing the incidence of CSA-AKl, measured with the standard creatinine technique and using neutrophil gelatinase-associated lipocalin (NGAL) serum concentrations as a potential new biomarker of kidney damage. The ethics committee of the Medical University of Lodz prospectively approved the protocol (approval No. RNN/286/13/KE). The study was retrospectively registered with the U.S. National Institutes of Health - NIH (29 June 2017; ClinicalTrials.gov identifier: NCT03205410).
\end{abstract}

Material and methods. We conducted a prospective single-center double-blind randomized and controlled tudy. Data was collected from patients admitted to the Cardiosurgery Clinic at the Medical University of Lodz (Poland) between January and December 2014, scheduled for elective cardiac surgery (an off-pump coronary artery bypass). A total of 28 patients were randomized to receive either RIPC $(n=14)$ or sham RIPC ( $n=14)$. After the induction of anesthesia, the patients assigned to the RIPC group underwent 3 cycles of five-minute inflation to $200 \mathrm{~mm} \mathrm{Hg}$ and five 5 -minute deflation of the upper-arm cuff. The control group had a deflated cuff placed on the upper arm for $30 \mathrm{~min}$. The authors measured the patients' serum creatinine concentration to check for the occurrence of a CSA-AKI within $48 \mathrm{~h}$ after cardiac surgery, and NGAL serum concentration to check its level within $3 \mathrm{~h}$ after the operation.

Results. Fewer patients in RIPC group developed CSA-AKI within $48 \mathrm{~h}$ after cardiac surgery than in the control group (29\% vs $93 \% ; p=0.003)$. Fewer patients in the RIPC group presented an increase in NGAL $3 \mathrm{~h}$ after surgery (medians: 124 vs $176.7 ; p=0.0003$ ).

Conclusions. In patients undergoing an off-pump coronary artery bypass, RIPC significantly reduces the occurrence of CSA-AKI and protects against increased postoperative NGAL levels.

Key words: neutrophil gelatinase-associated lipocalin, remote ischemic preconditioning, cardiac surgeryassociated acute kidney injury 
Acute kidney injury (AKI) is a well-known, serious complication and well-recognized independent risk factor of higher morbidity and mortality in patients undergoing cardiac surgery ${ }^{1,2}$; it is even referred to as cardiac surgeryassociated acute kidney injury (CSA-AKI). ${ }^{3}$ Approximately $30 \%$ of patients develop AKI after cardiac surgery ${ }^{4}$ and $1-5 \%$ of AKI patients require dialysis therapy. ${ }^{1,5}$ The CSAAKI can be caused by a variety of factors and in different combinations, including ischemia and reperfusion injury, toxins, metabolic abnormalities, neurohormonal activation, inflammation, and oxidative stress. ${ }^{6}$ Although preventing AKI after cardiac surgery would improve survival, there are still no efficient methods. ${ }^{7,8}$ The current definition of AKI is based on serum creatinine concentration ( $\mathrm{SCr}$ ) and urine output, and is described as any of the following: an increase in $\mathrm{SCr} \geq 0.3 \mathrm{mg} / \mathrm{dL}(\geq 26.5 \mathrm{lmol} / \mathrm{L})$ within $48 \mathrm{~h}$; or an increase in SCr to $\geq 1.5$ times baseline, which is known or presumed to have occurred within the prior 7 days; or urine volume $<0.5 \mathrm{~mL} / \mathrm{kg} / \mathrm{h}$ for 6 h. ${ }^{9}$ Both $\mathrm{SCr}$ and urine volume are markers of renal function but not kidney injury. ${ }^{10}$ Furthermore, according to the definition, AKI can be diagnosed using the creatinine technique after at least 2 days. This has led to investigations of new AKI biomarkers that could show kidney injury much earlier, within a few hours. During the past few decades several potential biomarkers of AKI have been identified, including neutrophil gelatinase-associated lipocalin (NGAL), ${ }^{11}$ kidney injury molecule 1 (KIM-1), ${ }^{12}$ interleukin 18 (IL-18), ${ }^{13}$ liver-type fatty acid-binding protein (L-FABP), ${ }^{14}$ tissue inhibitor of metalloproteinase 2 (TIMP-2), insulin-like growth factor-binding protein 7 (IGFBP7), ${ }^{15}$ calprotectin, ${ }^{16}$ and urine microRNAs. ${ }^{17}$ Neutrophil gelatinase-associated lipocalin is by far the most investigated and most promising, especially as an early AKI biomarker. Fast identification of AKI is very important, as is appropriate implementation of preventive strategies, which are the most effective tools to improve AKI outcome. ${ }^{18}$

Remote ischemic preconditioning (RIPC) is a phenomenon in which non-lethal periods of alternating ischemia and reperfusion applied to tissue or an organ can remotely protect another. At first, RIPC was known as a cardioprotection method, ${ }^{19}$ but it has also turned out to be effective in distant organs such as kidneys, offering protection in kidney transplantation ${ }^{20}$ or contrast-induced AKI, ${ }^{21}$ and seems promising in preventing AKI in patients who have undergone cardiac surgery. ${ }^{22,23}$ However, its efficacy still remains controversial. ${ }^{24}$ The mechanism of RIPC is complex and not well understood. Several triggers, intracellular pathways, humoral and neural effectors, as well as effectors induced by genetic changes may be considered potential pathways in the protective activity of RIPC. ${ }^{25}$

We conducted this prospective randomized controlled clinical study to assess whether RIPC reduces the incidence of AKI measured with the standard Scr technique and using neutrophil gelatinase-associated lipocalin (NGAL) as a potential new biomarker of kidney damage. The aim of our investigation was to analyze the safety and clinical outcomes of RIPC after elective isolated primary off-pump coronary artery bypass graft surgery (OPCAB).

\section{Material and methods}

\section{Study design}

This was a prospective single-center double-blind randomized controlled study. The ethics committee of the Medical University of Lodz (Poland) approved the protocol, and the study was conducted in accordance with the Helsinki Declaration and national law. Written informed consent was provided by all participants before enrollment in the study. The study design, along with the data collection and analysis, was conducted solely by the authors.

\section{The patients}

From January 2014 to December 2014, we screened patients over 18 years of age who were admitted to the Cardiosurgery Clinic of the Medical University of Lodz and scheduled for elective cardiac surgery (OPCAB). Enrollment was non-consecutive and dependent on whether one of the investigators who enrolled participants was available. Exclusion criteria were a history of cardiac surgery, acute myocardial infarction up to 7 days before surgery, chronic stage 4 or 5 kidney disease (estimated glomerular filtration rate $(\mathrm{eGFR})<30 \mathrm{~mL} / \mathrm{min} / 1.73 \mathrm{~m}^{2}$ ), peripheral vascular disease affecting the upper limbs, a history of severe injuries and operations within 2 months before cardiac surgery, a history of cancer, chronic autoimmune diseases, and dialysis. Patients were recruited during their preadmission consultations.

\section{Experimental protocol}

Following the placement of intravenous and right radial artery catheters and after the induction of anesthesia, the patients were randomly assigned in a 1:1 ratio to either the RIPC group or the control group by means of a computerized randomization table. A blinded investigator who was not involved in either the surgery or the randomization procedure performed RIPC in the RIPC group or sham RIPC in the control group. The RIPC group underwent 3 cycles of 5-minute inflation to $200 \mathrm{~mm} \mathrm{Hg}$ followed by 5 -minute deflation of the left upper-arm cuff (in excess of contralateral systolic radial artery pressure). The control group had a deflated cuff placed on the left upper arm for $30 \mathrm{~min}$. Remote ischemic preconditioning took place after the induction of anesthesia and was completed prior to skin incision.

\section{Surgical and anesthetic procedures}

Prescribed cardiac medications were administered up to the evening preceding surgery. Beta-adrenergic receptor 
antagonists were given on the day of the surgery, while agents that can interfere with RIPC (e.g., sulphonylurea, angiotensin-converting enzyme inhibitors, angiotensin receptor blockers) were transiently withdrawn $24 \mathrm{~h}$ before the operation. All the patients were given standardized nephroprotective procedures such as the withdrawal of potentially nephrotoxic agents $24 \mathrm{~h}$ before surgery and hydration by intravenous fluid infusion according to their clinical state, using the following formula: $60 \mathrm{~mL}$ of balanced solutions $+1 \mathrm{~mL}$ per every kilogram of body weight over $20 \mathrm{~kg}$ per hour (i.e., approx. 1.5-2 mL/ kg/h of balanced solutions) for $4 \mathrm{~h}$ prior to the surgery; and in patients with congestive heart failure or eGFR $<30 \mathrm{~mL} / \mathrm{min} / 1.73 \mathrm{~m}^{2}$ : infusion of $1 \mathrm{~mL} / \mathrm{kg} / \mathrm{h}$ of balanced solutions for $12 \mathrm{~h}$ prior to the surgery.

Anesthesia was induced with intravenous propofol $(1 \mathrm{mg} / \mathrm{kg})$, fentanyl $(3.5 \mu \mathrm{g} / \mathrm{kg})$ and pancuronium $(0.1 \mathrm{mg} / \mathrm{kg})$ and maintained with prolonged infusion of propofol (0.01-0.02 $\mathrm{mg} / \mathrm{kg} / \mathrm{min})$ and fentanyl $(0.05 \mu \mathrm{g} / \mathrm{kg} / \mathrm{min})$. All the patients were mechanically ventilated in controlled mechanical ventilation mode with $50 \%$ oxygen concentration. The surgical procedure was performed through median sternotomy according to standardized protocols. Postoperative fluid management in all the patients was performed according to clinical state of the individual patient and Enhanced Recovery After Surgery guidelines ${ }^{26}$ : $1.5 \mathrm{~mL} / \mathrm{kg} / \mathrm{h}$ on the day of the surgery, reduced the day after to $70 \mathrm{~mL} / \mathrm{h}$. Fluid therapy was conducted by monitoring central venous pressure, invasive blood pressure, pulse pressure variation, systolic pressure variation, urine volume, and the daily fluid balance. Serum osmolarity was maintained in the range of $280-305 \mathrm{mOsm} / \mathrm{kg} \mathrm{H} \mathrm{H}_{2} \mathrm{O}$. Fluid delivery included crystalloids (using balanced solutions excluding $0.9 \% \mathrm{NaCl}$ and $5 \%$ glucose $^{26}$ ). All of the participants were operated on by the same surgical team, and postoperative care was performed by the same anesthesiologist. The average duration of the surgery was $206 \mathrm{~min}$ in patients who received RIPC (median: $172.5 \mathrm{~min}$; interquartile range $(\mathrm{IQR})=155-260 \mathrm{~min})$ vs $187 \mathrm{~min}$ in the control group (median: $177.5 \mathrm{~min}$; IQR $=130-235 \mathrm{~min}$ ). The difference in duration between the 2 groups was not statistically significant $(\mathrm{p}=0.037)$.

\section{Blood sampling and analysis}

Venous blood samples were drawn before surgery and at $3 \mathrm{~h}$ and $48 \mathrm{~h}$ after surgery for measurement of serum creatinine and NGAL concentrations. Serum creatinine levels were measured with an enzymatic assay (Crea Creatinine OSR6578; Beckman Coulter Inc., Brea, USA). We used an enzyme-linked immunosorbent assay (ELISA) test to measure NGAL concentrations (Human Lipocalin - 2/NGAL ELISA; BioVendor Laboratory Medicine Inc., Brno-Řečkovice, Czech Republic). Estimated glomerular filtration rate was calculated using the Cockcroft-Gault formula.

\section{Endpoints}

The primary endpoint of the study was the incidence of AKI within $48 \mathrm{~h}$ after cardiac surgery or increased NGAL level within $3 \mathrm{~h}$ after the operation. Acute kidney injury was classified according to the Kidney Disease Improving Global Outcomes (KDIGO) criteria as any of the following: (1) an increase in $\mathrm{SCr} \geq 0.3 \mathrm{mg} / \mathrm{dL}(\geq 26.5 \mathrm{lmol} / \mathrm{L})$ within $48 \mathrm{~h}$ after surgery; or (2) an increase in SCr to $\geq 1.5$ times baseline, which is known or presumed to have occurred within the previous 7 days; or (3) urine volume $<0.5 \mathrm{~mL} / \mathrm{kg} / \mathrm{h}$ for $6 \mathrm{~h}$ after surgery.

Secondary endpoints were the length of hospitalization, the length of intensive care unit (ICU) stay, ventilation time, the occurrence of postoperative atrial fibrillation (AF), the time of renal replacement therapy (RRT), and death from any cause.

\section{Statistical analysis}

We performed the statistical analysis using the STATISTICA v. 10 software (StatSoft Polska, Kraków, Poland). For all the tests, we used $\mathrm{p}=0.05$ as the threshold of statistical significance. The Shapiro-Wilk normality test was used to verify the distribution assumptions for normality. Categorical variables are represented as the number of observations $(\mathrm{N})$ and the corresponding percentages (\%). Quantitative variables are presented as median and IQR. Pearson's $X^{2}$ test was used to check group equality. If the number of cases was less than 5, Yates's correction for continuity was used. The distribution of most of the variables under consideration was not normal. Continuous variables that were not distributed normally were analyzed with a nonparametric test. In order to compare 2 independent trials, the Mann-Whitney U test was used. For a comparison of 2 repeated measurements between 2 matched samples of continuous variables, we used the Wilcoxon signedrank test. To detect differences in continuous values across multiple test attempts, we used Friedman's test.

\section{Results}

\section{Study population characteristics and operative data}

A total of 58 patients were assessed for eligibility, with 30 patients excluded before randomization due to an exclusion criteria or consent withdrawal. This left 28 patients who were enrolled and randomized to receive either RIPC ( $\mathrm{n}=14$ ) or sham RIPC ( $=14)$ and included in the primary analysis (Fig. 1). The preoperative characteristics and intraoperative protocols were similar in the 2 groups (Table 1). Preoperative serum creatinine and NGAL concentrations were also similar in the 2 groups. The time between the end of the last inflation of the blood-pressure cuff and the skin incision was $6 \pm 1 \mathrm{~min}$. 
Table 1. Characteristics of the study population

\begin{tabular}{|c|c|c|c|}
\hline Characteristics & $\operatorname{RIPC}(n=14)$ & Control $(n=14)$ & $p$-value \\
\hline Age [years] & $65(60-71)$ & $67(61-72)$ & 0.804 \\
\hline $\operatorname{Sex}(\%)$ & $\begin{array}{l}\text { M: } 9(64) \\
F: 6(36)\end{array}$ & $\begin{array}{c}M: 8(57) \\
F: 6(43)\end{array}$ & 0.699 \\
\hline BMI $\left[\mathrm{kg} / \mathrm{m}^{2}\right]$ & $31(29.1-32.3)$ & $27.7(25.4-30.9)$ & 0.062 \\
\hline Systolic blood pressure [mm Hg] & $112.5(105-120)$ & $120(120-130)$ & 0.077 \\
\hline Diastolic blood pressure [mm Hg] & $70(65-70)$ & $70(70-80)$ & 0.164 \\
\hline $\mathrm{GFR}\left[\mathrm{mL} / \mathrm{min} / 1.73 \mathrm{~m}^{2}\right]$ & $108(82-118)$ & $73.5(55-105)$ & 0.062 \\
\hline $\mathrm{SCr}[\mu \mathrm{mol} / \mathrm{L}]$ & $72.5(61-82)$ & 85.5 (69-108) & 0.21 \\
\hline Serum NGAL concentration [ng/mL] & $127.1(102.3-139.5)$ & $102.3(68.2-139.5)$ & 0.227 \\
\hline CCS class, n (\%) & $2.5(2-3)$ & $2(2-2)$ & 0.227 \\
\hline NYHA class, n (\%) & $2(2-3)$ & $2.5(2-3)$ & 0.839 \\
\hline History of heart attack, n (\%) & $7(50)$ & $7(50)$ & 1 \\
\hline History of stroke/transient ischemic attack, n (\%) & $2(14)$ & $0(0)$ & 0.463 \\
\hline Current smoking, $n(\%)$ & $9(64)$ & $7(50)$ & 0.445 \\
\hline Hypertension arterialis, n (\%) & $14(100)$ & $13(93)$ & 0.309 \\
\hline Chronic heart failure, n (\%) & $4(29)$ & $4(29)$ & 0.676 \\
\hline Dyslipidemia, n (\%) & $11(79)$ & $12(86)$ & 0.622 \\
\hline IGT/IFG, n (\%) & $0(0)$ & $1(7)$ & 1.00 \\
\hline Diabetes mellitus, n (\%) & $5(36)$ & $8(57)$ & 0.256 \\
\hline COPD, n (\%) & $1(7)$ & $3(21)$ & 0.59 \\
\hline Chronic kidney disease, n (\%) & $1(7)$ & $2(14)$ & 1.00 \\
\hline Nephrolithiasis, n (\%) & $1(7)$ & $2(14)$ & 1.00 \\
\hline
\end{tabular}

Data is presented as median (interquartile range) or $\mathrm{n}$ (\%). M - male; F - female; RIPC - remote ischemic preconditioning; BMI - body mass index; GFR - glomerular filtration rate; SCr - serum creatinine concentration; NGAL - neutrophil gelatinase-associated lipocalin; CCS - Canadian Cardiovascular Society; NYHA - New York Heart Association; IGT - impaired glucose tolerance; IFG - impaired fasting glucose; COPD - chronic obstructive pulmonary disease.

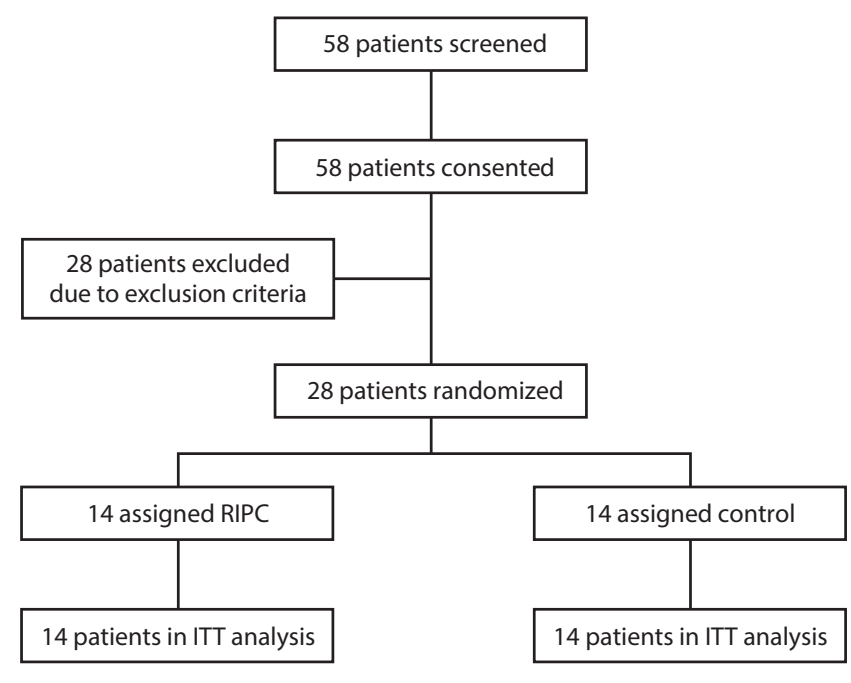

Fig. 1. Flowchart of the patients in the study

RIPC - remote ischemic preconditioning; ITT - intention-to-treat analysis.

\section{Primary outcomes}

Significantly fewer patients in the RIPC group developed AKI within $48 \mathrm{~h}$ after cardiac surgery compared with the control group (Table 2), with absolute risk reduction of 0.64. Similarly, the patients in the RIPC group presented significantly lower serum NGAL concentrations $3 \mathrm{~h}$ after surgery compared to the control group (Table 2). Moreover, the patients who received RIPC showed either a decrease or only a slight increase in serum NGAL levels compared to the control group, who manifested significant increases in NGAL levels (Fig. 2).

\section{Secondary outcomes}

Serum creatinine concentration ( $\mathrm{SCr}$ ), tested on admission, did not differ between the 2 groups $(\mathrm{p}=0.21$; Table 1$)$. However, analysis of SCr over time - on admission, $48 \mathrm{~h}$ after OPCAB and on discharge - showed that in the patients who received RIPC before cardiac surgery, $\mathrm{SCr}$ did not change statistically ( $\mathrm{p}=0.147$; Fig. 3 ). In contrast, the patients in the control group showed significantly different levels of SCr over time ( $\mathrm{p}=0.0004$; Fig. 3). Likewise, GFR was not significantly different in the 2 groups at baseline $(\mathrm{p}=0.062 ;$ Table 1). Glomerular filtration rate changes over time were not significantly different in the RIPC group as opposed to the control group ( $\mathrm{p}=0.374$ vs $\mathrm{p}=0.0499$; Fig. 4). However, we found no significant differences between the groups in terms of the time of receiving mechanical ventilation $(p=0.756)$, the length of their stay 
Table 2. Operative and postoperative history of the study participants

\begin{tabular}{|c|c|c|c|}
\hline Variable & RIPC & Control & p-value \\
\hline Occurrence of AKI (\%) & $4(29)$ & $13(93)$ & 0.003 \\
\hline GFR on admission $\left[\mathrm{mL} / \mathrm{min} / 1.73 \mathrm{~m}^{2}\right]$ & $108(82-118)$ & $73.5(55-105)$ & 0.062 \\
\hline GFR $48 \mathrm{~h}$ after surgery $\left[\mathrm{mL} / \mathrm{min} / 1.73 \mathrm{~m}^{2}\right]$ & $95.5(67-137)$ & $47.5(36-69)$ & 0.005 \\
\hline GFR on discharge $\left[\mathrm{mL} / \mathrm{min} / 1.73 \mathrm{~m}^{2}\right]$ & $98.5(81-141)$ & $72(70-107)$ & 0.062 \\
\hline SCr on admission $[\mu \mathrm{mol} / \mathrm{L}]$ & $72.5(61-82)$ & $85.5(69-108)$ & 0.21 \\
\hline $\mathrm{SCr} 48 \mathrm{~h}$ after surgery $[\mu \mathrm{mol} / \mathrm{L}]$ & $79.5(70-125)$ & $130.5(102-158)$ & 0.014 \\
\hline $\mathrm{SCr}$ on discharge $[\mu \mathrm{mol} / \mathrm{L}]$ & $70(60-84)$ & $75(71-93)$ & 0.454 \\
\hline NGAL on admission [ng/mL] & $127.1(102.3-139.5)$ & $102.3(68.2-139.5)$ & 0.227 \\
\hline NGAL $3 \mathrm{~h}$ after surgery [ng/mL] & $124(111.6-142.6)$ & $176.7(155.0-204.6)$ & 0.0003 \\
\hline Hospitalization [days] & $11(10-14)$ & $10(9-13)$ & 0.454 \\
\hline ICU stay [days] & $2.5(2-8)$ & $3(2-3)$ & 0.667 \\
\hline Ventilation time [days] & $1(1-1)$ & $1(1-1)$ & 0.756 \\
\hline Time of RRT [days] & $0(0-0)$ & $0(0-0)$ & 0.982 \\
\hline Postoperative AF (\%) & $2(14)$ & $5(36)$ & 0.383 \\
\hline Death (\%) & $1(7)$ & $0(0)$ & 1.00 \\
\hline
\end{tabular}

Data is presented as median (interquartile range) or n (\%). RIPC - remote ischemic preconditioning; AKI - acute kidney injury; GFR - glomerular filtration rate; $\mathrm{SCr}$ - serum creatinine concentration; NGAL - neutrophil gelatinase-associated lipocalin; ICU - intensive care unit; RRT - renal replacement therapy; AF - atrial fibrillation.

A

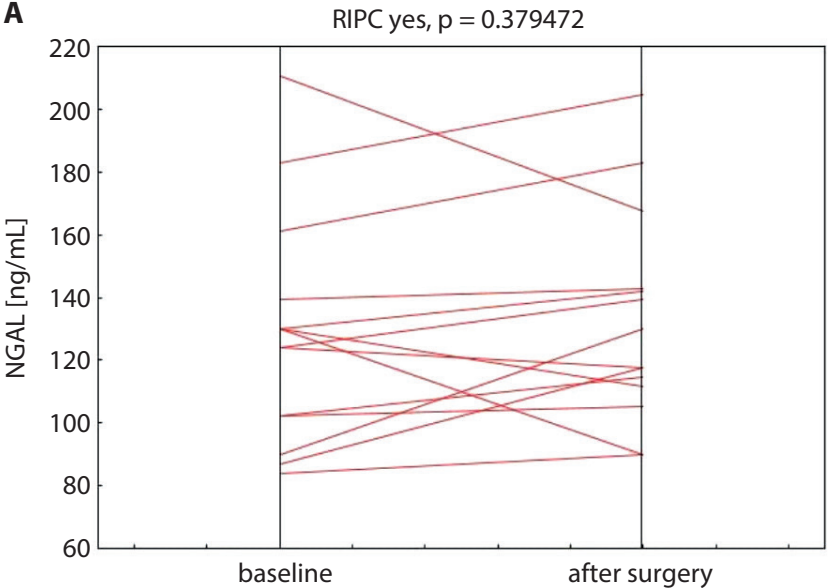

B

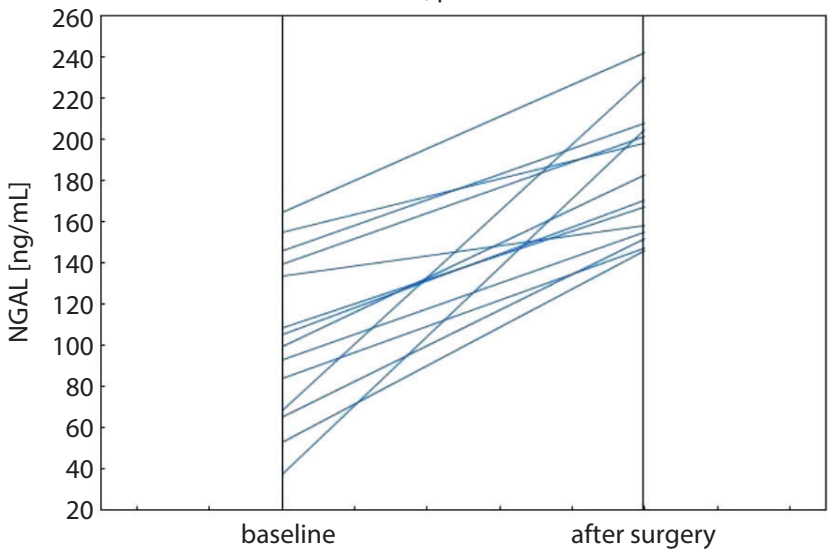

Fig. 2. NGAL serum concentration increase (difference between baseline and $3 \mathrm{~h}$ after surgery) in (A) the patients who received RIPC and (B) the control group (no RIPC)

NGAL - neutrophil gelatinase-associated lipocalin; RIPC - remote ischemic preconditioning.

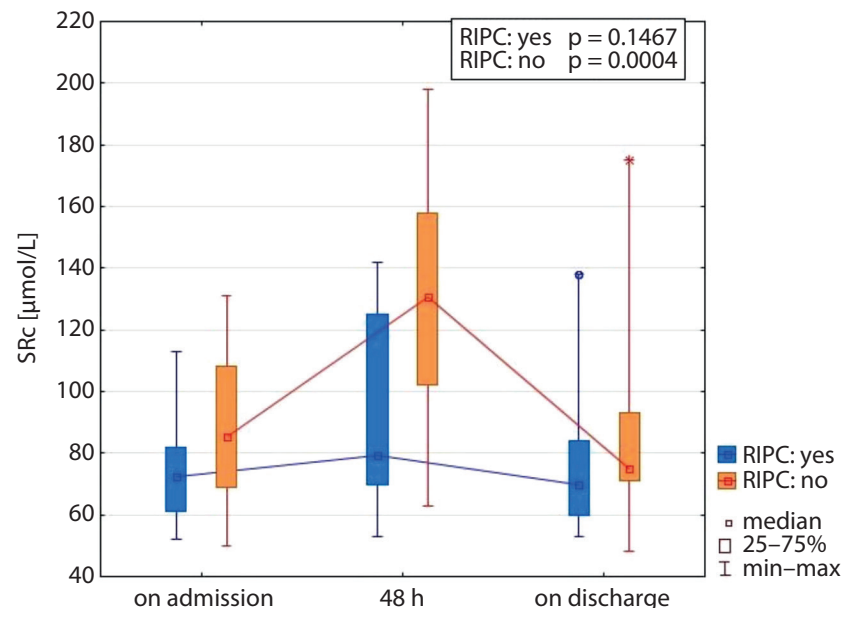

Fig. 3. Variability of serum creatinine concentration [ $\mu \mathrm{mol} / \mathrm{L}]$ over time (admission to the Cardiosurgery Clinic, $48 \mathrm{~h}$ after surgery, discharge from the hospital) in the RIPC group vs the control group (no RIPC)

$\mathrm{SCr}$ - serum creatinine concentration; RIPC - remote ischemic preconditioning.

in the ICU ( $p=0.667)$, the length of their hospital stay $(\mathrm{p}=0.454)$, the occurrence of postoperative AF ( $\mathrm{p}=0.383)$, or death $(\mathrm{p}=1.00)$. In each group, 1 patient required dialysis in the postoperative period, and there were no significant differences in the length of renal replacement therapy $(\mathrm{p}=0.982)$.

\section{Discussion}

Cardiac surgery patients have a high risk of AKI. Simultaneously, the development of AKI is associated with higher mortality and a higher risk of complications in patients 


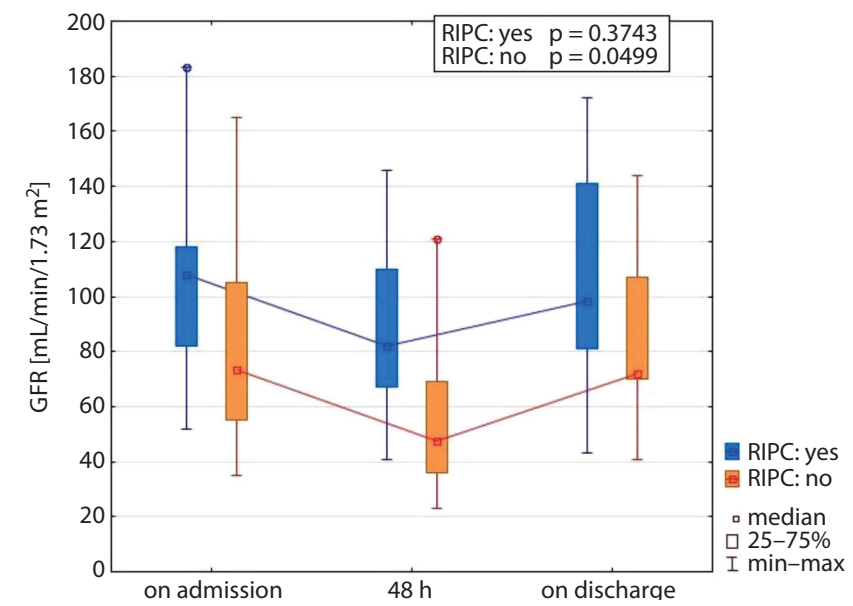

Fig. 4. Variability of GFR [mL/min/1.73 $\left.\mathrm{m}^{2}\right]$ over time (admission

to the Cardiosurgery Clinic, $48 \mathrm{~h}$ after surgery, discharge from the hospital) in (1) the RIPC group vs (0) the control (no RIPC) group

eGFR - estimated glomerular filtration rate; RIPC - remote ischemic preconditioning.

undergoing cardiac surgery. ${ }^{27}$ However, there are no effective clinical strategies for preventing AKI. Remote ischemic preconditioning holds promise as a simple and inexpensive way of protecting tissues against ischemic damage, including kidney protection, which has led to research into the use of this method to prevent AKI. Nowadays, the standard diagnostic tools for AKI detection, such as $\mathrm{SCr}$ and urine output monitoring, are markers of renal function but not kidney injury. Furthermore, SCr depends on various intrarenal and extrarenal functions and its concentration characterizes the balance between creatinine generation and excretion. ${ }^{28}$ Serum creatinine concentration is a delayed and insensitive biomarker of changes in kidney function, and its concentration does not differentiate the triggers of kidney failure and could be affected by many factors. ${ }^{29}$ Damage biomarker such as NGAL may quickly allow cellular kidney damage to be identified and lead to earlier diagnosis of AKI. Although NGAL is represented in some human tissues, it is one of the most upregulated transcripts in the kidney after ischemic, toxic or septic AKI in animal and human models, implying that it has a role as an early marker of structural renal tubular damage. ${ }^{30}$

In our single-center double-blind study involving 28 patients at a high risk of postoperative AKI, RIPC did reduce the prevalence of $\mathrm{AKI}$, according to KDIGO criteria, based on increases in SCr. Our surprising finding that $93 \%$ of the control group as well as $29 \%$ of the RIPC group developed AKI may result from the small number of participants as well as the sensitivity of the KDIGO AKI definition, which is based on only a slight elevation in SCr. Furthermore, we showed the benefit of RIPC, with reduced levels of SCr and higher GFR $48 \mathrm{~h}$ after OPCAB. In the patients who received RIPC prior to surgery, only a $9.66 \%$ increase in SCr level compared to the baseline was observed.
In contrast, in the patients without RIPC, the postoperative SCr level increased significantly, by as much as $52.63 \%$. Remote ischemic preconditioning turned out to be protective against significant increases in $\mathrm{SCr}$ as well as decreases in GFR over time. Moreover, we found that the postoperative expression of NGAL, an early biomarker of AKI, was significantly reduced in patients who underwent RIPC.

Even though the prevalence of AKI was lower in the RIPC group, our study found no benefits of RIPC in terms of the length of ICU stay, the duration of mechanical ventilation or length of hospitalization. This may be due to the small study group. Although fewer patients in the RIPC group showed postoperative AF, the overall assessment showed no significant differences.

The effect of RIPC on kidney function differs among studies. Our findings are consistent with the randomized controlled trial by Zarbock et al. ${ }^{31}$ Their study was specifically designed and powered to look at the effect of RIPC on AKI as the primary endpoint. As in our research, they noticed a significant absolute risk reduction in the incidence of AKI in the RIPC group, and higher postoperative NGAL levels in the control group $(\mathrm{p}=0.04)$. Furthermore, in a meta-analysis including 26 trials, the rate of AKI was significantly lower in the RIPC groups than in the control groups among patients undergoing cardiac and vascular interventions $(\mathrm{p}=0.001$; $R \mathrm{R}=0.79) .{ }^{32}$ However, it should be noticed that various definitions of AKI were used in different studies. The same report found no benefits of RIPC in postoperative $\mathrm{SCr}$ and eGFR levels, in-hospital mortality, initiation of RRT, or the length of hospital stay. This is consistent with a similar meta-analysis where postoperative incidence of AKI was significantly reduced by RIPC $(\mathrm{p}=0.02)$, but no benefit was found in terms of renal replacement therapy and mortality. ${ }^{33}$ A recent meta-analysis including 27 randomized trials also showed that RIPC lowers the risk not only of acute renal failure, but also myocardial infarction, stroke and composite risk of allcause mortality; however, statistically the results were only marginally significant. ${ }^{34}$ The recently published results of the 90-day follow-up of the RenalRIP trial showed that RIPC improves short- as well as long-term outcomes of high-risk patients undergoing cardiac surgery. ${ }^{35}$ In that study, RIPC clearly reduced the occurrence of major adverse kidney events at 90 days (including all-cause mortality, RRT and persistent renal dysfunction without dialysis), compared with the controls. Also, considering different components of composite endpoints, persistent renal dysfunction and RRT were significantly higher in the patients that did not undergo RIPC. ${ }^{35}$

On the other hand, some trials reported that RIPC did not lead to any significant difference in clinical outcomes compared to the controls. In an 11-center randomized controlled trial involving patients at high risk of AKI and undergoing cardiac surgery, RIPC yielded no demonstrable benefits. The median peak of postoperative change in creatinine was not statistically significant (absolute 
mean difference: $0.06,95 \%$ confidence interval (95\% CI) $=0.10-0.23) \cdot{ }^{36}$ Likewise, in the RIPValve study, in patients with aortic valve stenosis who underwent elective aortic valve replacement, RIPC also had no impact on postoperative renal function. ${ }^{37}$ Two large multicenter doubleblind randomized controlled trials where propofol was used to maintain anesthesia noted no benefits of RIPC. The Remote Ischemic Preconditioning for Heart Surgery (RIPHeart) study and the Effect of Remote Ischemic Preconditioning on Clinical Outcomes in Patients Undergoing Cardiac Surgery (ERICCA) study investigated clinical outcomes in patients undergoing cardiac surgery. ${ }^{38,39}$ Neither of them showed any evidence of positive effects of RIPC on death within 12 months, postoperative AF, AKI, postoperative release of NGAL, or the duration of ICU and hospital stay. ${ }^{40}$ The use of propofol anesthesia in more than $90 \%$ of the patients of ERICCA and all the patients in RIPHeart is the most plausible explanation for the failure of RIPC to provide protection. ${ }^{41}$

Similarly, the presence of diabetes mellitus may impair conditioning-mediated protection. ${ }^{42}$ Despite the fact that $36 \%$ of the RIPC group and $57 \%$ of the control group in our study presented diabetes mellitus, we found that RIPC protected against the development of CSA-AKI. Possible explanations for the differences in findings may include differences in the patient populations, the duration of RIPC and, of course, the small sample size in our study.

\section{Limitations}

Our study has some limitations. It is a single-center trial with a relatively small sample size, and although we have found important associations with intermediary endpoints, we cannot prove the mechanism. Also, enrollment in the study depended upon the availability of the investigator, which could have biased the sample. It may also have contributed to our surprising finding that $93 \%$ of the control group and 29\% of the RIPC group developed AKI, which distinguishes our study from the literature. Possible explanations for these differences may be the small sample size of our study, but also the fact that GFR at admission is almost significantly lower $(p=0.062)$ in the control patients compare to the patients enrolled in the RIPC group.

\section{Conclusions}

In patients undergoing OPCAB, RIPC significantly reduces the occurrence of CSI-AKI and limits SCr increase over time. The extremely easy-to-apply, low-cost and non-invasive nature of RIPC makes it an ideal method for the prevention of AKI. The introduction of RIPC strategy into widespread clinical settings for the benefit of patients undergoing heart surgery could represent a promising and simple strategy to provide additional protection of kidney function and improve postoperative outcomes.
Remote ischemic preconditioning may become "the future of nephroprotection" in cardiac surgery. The same applies to the RIPC-mediated postoperative NGAL reduction noted in our pilot trial. Neutrophil gelatinase-associated lipocalin is one of the best biomarkers of AKI, due to its quick release after tubular damage. It opens a new era of earlier detection and prognosis prediction for AKI, compared to the standard definition. It also creates an urgent need to come to an agreement about the cutoff value of NGAL, which should help in redefining AKI according to NGAL levels. Apart from its limitations, our study demonstrated the important role RIPC plays in protecting against AKI after cardiac surgery. Hence, further studies are needed to redefine the clinical utility of RIPC in current practice and to obtain more evidence of its potential benefits.

\section{ORCID iDs}

Karolina Stokfisz (D) https://orcid.org/0000-0002-0908-3477 Anna Ledakowicz-Polak (D) https://orcid.org/0000-0003-3355-4061 Maciej Zagórski (D) https://orcid.org/0000-0003-4999-4491 Sławomir Jander (D) https://orcid.org/0000-0002-5565-8796 Katarzyna Przybylak (D) https://orcid.org/0000-0003-3241-2483 Marzenna Zielińska (D) https://orcid.org/0000-0002-0118-8610

\section{References}

1. Thakar CV, Worley S, Arrigain S, Yared JP, Paganini EP. Improved survival in acute kidney injury after cardiac surgery. Am J Kidney Dis. 2007;50(5):703-711.

2. Loef BG, Epema AH, Navis G, Ebels T, Stegeman CA. Postoperative renal dysfunction and preoperative left ventricular dysfunction predispose patients to increased long-term mortality after coronary artery bypass graft surgery. Br J Anaesth. 2009;102(6):749-755. doi:10. 1093/bja/aep088

3. Vandenberghe W, De Loor J, Hoste EA. Diagnosis of cardiac surgeryassociated acute kidney injury from functional to damage biomarkers. Curr Opin Anaesthesiol. 2017;30(1):66-75.

4. Rosner MH, Okusa MD. Acute kidney injury associated with cardiac surgery. Clin J Am Soc Nephrol. 2006;1(1):19-32.

5. Ostermann ME, Taube D, Morgan CJ, Evans TW. Acute renal failure following cardiopulmonary bypass: A changing picture. Intensive Care Med. 2000;26(5):565-571.

6. Bellomo R, Auriemma S, Fabbri A, et al. The pathophysiology of cardiac surgery-associated acute kidney injury (CSA-AKI). Int J Artif Organs. 2008;31(2):166-178.

7. Haase $M$, Haase-Fielitz A, Plass $M$, et al. Prophylactic perioperative sodium bicarbonate to prevent acute kidney injury following open heart surgery: A multicenter double-blinded randomized controlled trial.PLoSMed.2013;10(4):e1001426. doi:10.1371/journal.pmed.1001426

8. Lameire N, van Biesen W, Hoste E, Vanholder R. The prevention of acute kidney injury: An in-depth narrative review. Part 2: Drugs in the prevention of acute kidney injury. NDT Plus. 2009;2(1):1-10. doi:10. 1093/ndtplus/sfn199

9. KDIGO AKI Work Group. KDIGO clinical practice guideline for acute kidney injury. Kidney Int Suppl. 2012;2:1-138.

10. Waikar SS, Betensky RA, Bonventre JV. Creatinine as the gold standard for kidney injury biomarker studies? Nephrol Dial Transplant. 2009;24(11):3263-3265. doi:10.1093/ndt/gfp428

11. Zhou F, Luo Q, Wang L, Han L. Diagnostic value of neutrophil gelatinase-associated lipocalin for early diagnosis of cardiac surgery-associated acute kidney injury: A meta-analysis. Eur J Cardiothorac Surg. 2016;49(3):746-755.

12. Shao $X, \operatorname{Tian} L, X u W$, et al. Diagnostic value of urinary kidney injury molecule 1 for acute kidney injury: A meta-analysis. PLoS One. 2014; 9(1):e84131.

13. Lin X, Yuan J, Zhao Y, Zha Y. Urine interleukin-18 in prediction of acute kidney injury: A systemic review and meta-analysis. J Nephrol. 2015; 28:7-16. 
14. Xu Y, Xie Y, Shao X, Ni Z, Mou S. L-FABP: A novel biomarker of kidney disease. Clin Chim Acta. 2015;445:85-90.

15. Kashani K, Al-Khafaji A, Ardiles T, et al. Discovery and validation of cell cycle arrest biomarkers in human acute kidney injury. Crit Care. 2013;17(1):R25. doi:10.1186/cc12503

16. Heller F, Frischmann S, Grunbaum M, Zidek W, Westhoff TH. Urinary calprotectin and the distinction between prerenal and intrinsic acute kidney injury. Clin J Am Soc Nephrol. 2011;6(10):2347-2355.

17. Lorenzen JM, Kielstein JT, Hafer C, et al. Circulating miR-210 predicts survival in critically ill patients with acute kidney injury. Clin J Am Soc Nephrol. 2011;6(7):1540-1546. doi:10.2215/CJN.00430111

18. Cruz DN, Bagshaw SM, Maisel A, et al. Use of biomarkers to assess prognosis and guide management of patients with acute kidney injury. Contrib Nephrol. 2013;182:45-64. doi:10.1159/000349965

19. Przyklenk K, Bauer B, Ovize M, Kloner RA, Whittaker P. Regional ischemic 'preconditioning' protects remote virgin myocardium from subsequent sustained coronary occlusion. Circulation. 199;87(3):893-899.

20. Veighey K, MacAllister R. Ischemic conditioning in kidney transplantation. J Cardiovasc Pharmacol Ther. 2017;22(4):330-336. doi:10.1177/ 1074248417702893

21. Er F, Nia AM, Dopp H, et al. Ischemic preconditioning for prevention of contrast medium-induced nephropathy: Randomized pilot RenPro Trial (Renal Protection Trial). Circulation. 2012;126(3):296-303. doi:10.1161/CIRCULATIONAHA.112.096370

22. Zimmerman RF, Ezeanuna PU, Kane JC, et al. Ischemic preconditioning at a remote site prevents acute kidney injury in patients following cardiac surgery. Kidney Int. 2011;80(8):861-867.

23. Ali ZA, Callaghan CJ, Lim E, et al. Remote ischemic preconditioning reduces myocardial and renal injury after elective abdominal aortic aneurysm repair: A randomized controlled trial. Circulation. 2007; 116(11 Suppl):198-105.

24. Giannopoulos G, Vrachatis DA, Panagopoulou V, Vavuranakis M, Cleman MW, Deftereos S. Remote ischemic conditioning and renal protection. J Cardiovasc Pharmacol Ther. 2017;22(4):321-329. doi:10. $1177 / 1074248417702480$

25. Stokfisz K, Ledakowicz-Polak A, Zagorski M, Zielinska M. Ischaemic preconditioning: Current knowledge and potential future applications after 30 years of experience. Adv Med Sci. 2017;62(2):307-316. doi:10.1016/j.advms.2016.11.006

26. Miller TE, Roche AM, Mythen M. Fluid management and goal-directed therapy as an adjunct to Enhanced Recovery After Surgery (ERAS). Can J Anaesth. 2015;62(2):158-168. doi:10.1007/s12630-014-0266-y

27. Conlon PJ, Crowley J, Stack R, et al. Renal artery stenosis is not associated with the development of acute renal failure following coronary artery bypass grafting. Ren Fail. 2005;27(1):81-86

28. Au V, Feit J, Barasch J, Sladen RN, Wagener G. Urinary neutrophil gelatinase-associated lipocalin (NGAL) distinguishes sustained from transient acute kidney injury after general surgery. Kidney Int Rep. 2016;1(1):3-9.

29. Kashani K, Cheungpasitporn W, Ronco C. Biomarkers of acute kidney injury: The pathway from discovery to clinical adoption. Clin Chem Lab Med. 2017;55(8):1074-1089.
30. Mishra J, Mori K, Ma Q, Kelly C, Barasch J, Devarajan P. Neutrophil gelatinase-associated lipocalin: A novel early urinary biomarker for cisplatin nephrotoxicity. Am J Nephrol. 2004;24(3):307-315.

31. Zarbock A, Schmidt C, Van Aken H, et al; RenalRIPC Investigators. Effect of remote ischemic preconditioning on kidney injury among high-risk patients undergoing cardiac surgery: A randomized clinical trial. JAMA. 2015;313(21):2133-2141. doi:10.1001/jama.2015.4189

32. Li B, Lang $X, C a o ~ L$, et al. Effect of remote ischemic preconditioning on postoperative acute kidney injury among patients undergoing cardiac and vascular interventions: A meta-analysis. J Nephrol. 2017; 30(1):19-33. doi:10.1007/s40620-016-0301-x

33. Zhou C, Bulluck H, Fang N, Li L, Hausenloy DJ. Age and surgical complexity impact on renoprotection by remote ischemic preconditioning during adult cardiac surgery: A meta-analysis. SciRep. 2017;7(1):215. doi:10.1038/s41598-017-00308-3

34. Sardar $\mathrm{P}$, Chatterjee $\mathrm{S}$, Kundu $\mathrm{A}$, et al. Remote ischemic preconditioning in patients undergoing cardiovascular surgery: Evidence from a meta-analysis of randomized controlled trials. Int J Cardiol. 2016; 221:34-41. doi:10.1016/j.ijcard.2016.06.325

35. Zarbock A, Kellum JA, Van Aken $\mathrm{H}$, et al. Long-term effects of remote ischemic preconditioning on kidney function in high-risk cardiac surgery patients: Follow-up results from the RenalRIPTrial. Anesthesiology. 2017:126(5):787-798. doi:10.1097/ALN.0000000000001598

36. Walsh M, Whitlock R, Garg AX, et al; Remote IMPACT Investigators. Effects of remote ischemic preconditioning in high-risk patients undergoing cardiac surgery (Remote IMPACT): A randomized controlled trial. CMAJ. 2016;188(5):329-336. doi:10.1503/cmaj.150632

37. Pinaud F, Corbeau JJ, Baufreton C, et al. Remote ischemic preconditioning in aortic valve surgery: Results of a randomized controlled study. J Cardiol. 2016;67(1):36-41. doi:0.1016/j.jjcc.2015.06.007

38. Hausenloy DJ, Candilio L, Laing C, et al; ERICCA Trial Investigators. Effect of remote ischemic preconditioning on clinical outcomes in patients undergoing coronary artery bypass graft surgery (ERICCA): Rationale and study design of a multi-centre randomized doubleblinded controlled clinical trial. Clin Res Cardiol. 2012;101(5):339-348.

39. Meybohm P, Bein B, Brosteanu O, et al; RIPHeart Study Collaborators. A multicenter trial of remote ischemic preconditioning for heart surgery. N Engl J Med. 2015;373(15):1397-1407.

40. Garratt KN, Whittaker P, Przyklenk K. Remote ischemic conditioning and the long road to clinical translation: Lessons learned from ERICCA and RIPHeart. Circ Res. 2016;118(7):1052-1054. doi:10.1161/CIRCRESAHA.115.308102

41. Heusch G, Gersh BJ. ERICCA and RIPHeart: Two nails in the coffin for cardioprotection by remote ischemic conditioning? Probably not! Eur Heart J. 2016;37(2):200-202. doi:10.1093/eurheartj/ehv606

42. Przyklenk K. Efficacy of cardioprotective 'conditioning' strategies in aging and diabetic cohorts: The co-morbidity conundrum. Drugs Aging. 2011;28(5):331-343. doi:10.2165/11587190-000000000-00000 